\title{
2.5-D GEOMETRICAL SPREADING IN A LAYERED ISOTROPIC MEDIUM UNDER DEEP-WATER CONDITION
}

\author{
LIU, M. C. - SUN, J. G. ${ }^{*}-$ HAN, F. X. - LIU, Z. Q. ${ }^{*}-$ SUN, H. \\ College for Geoexploration Science and Technology, Jilin University \\ No.938, Ximinzhu Street, Changchun, Jilin Province, 130026, P. R. China \\ (phone: +04-31-8592-3862; fax: +04-31-8592-3862) \\ ${ }^{*}$ Corresponding authors \\ e-mail:sun_jg@jlu.edu.cn; liuzq13@mails.jlu.edu.cn; Sun_hui_jlu@163.com
}

(Received 22 $2^{\text {nd }}$ Jul 2016; accepted $9^{\text {th }}$ Nov 2016)

\begin{abstract}
Proceeding from the perspective of Physical Oceanography, we study the theoretical models and analyze the influence of marine channel and offset on the geometrical spreading compensation value. Concerning the long offset and deep water conditions during the geophysical exploration process, we put forward the 2.5-D geometrical spreading compensation technique to conduct more accurate amplitude restoration of the horizontal layered or seemingly horizontal layered medium. The method fully considers the geometrical spreading compensation and the marine channel outside the plane in the 2-D observation system to achieve optimal restoration of the amplitude. 2.5-D has small computational amount that can meet the real-time seismic processing requirement. The energy equilibrium analysis, synthetic and field data results suggest that the method can ensure the equilibrium of the seismic information energy of different underwater depths more properly, and improve the information reliability.
\end{abstract}

Keywords: marine channel, seismology, seawater velocities, energy equilibrium analysis, data analysis

\section{Introduction}

Geometrical spreading refers to the continuous spreading of the amplitude energy along with the increase of the spreading distance during the underground spreading of the seismic wave. The divergent process of the seismic wave is unavoidable, so the knowledge of how to quickly and accurately conduct amplitude compensation is of vital importance.

The traditional geometrical spreading compensatiohn theoretic researches mainly focused on the geometrical spreading compensation of the land layered medium. In view of the research status, Newman (1973) inferred the zero shot-offset geometrical spreading compensation formula. It is a convenient and simple method, which has found wide applications in real life, but its compensation accuracy is limited. Ursin et al. (1978, 1990, 1992, 2003, 2006) systematically studied the relationship among seismic ray tracing, travel time and amplitude compensation. Several approximate formulas to calculate the layered medium under the condition of non-zero shot-offset. To get better approximate effect, it is necessary to learn the high-order time average velocity. The speed is got through inversion, thus causing great uncertainty to results. In 1986, Bleistein published the famous 2.5-D theory, pointing out that 2.5-D is the spreading rule of the point source in the 2-D plane. It is a theory aiming at achieving an approximate effect of 3-D through items within and outside the plane. The significance of 2.5-D theory lies in its approximation to 3-D practical conditions with less computational amount required of 3-D so as to meet the real-time seismic processing requirement and lay theoretical foundation for 2.5-D geometrical spreading compensation. Later, many scholars (Červený and Ravindra, 1971; Bortfeld, 1989; Tygel et al., 1992; Sun, 1996; Červený, 2001; Stovas et al., 2009) studied different 
aspects of geometrical spreading. In 2002, Ettrich studied the issue of geometrical spreading compensation under the marine condition, analyzing the compensation curve with the seawater velocity as constant as $1,500 \mathrm{~m} / \mathrm{s}$. However, in that article, the influence of the marine channel on geometrical spreading was not considered. Generally speaking, to conduct survey of the subsea oil and gas under the condition of the constant seawater velocity will cause significant errors. Research (Han et al., 2012) suggested that marine channel in the seawater exert a considerable influence on the seismic ray path, travel time and amplitude. Quite often, the deeper seawater has an more obvious influence on geometrical spreading. While the conventional compensation style does not take underwater channels into consideration, it will impair the accuracy of the geometrical spreading compensation. Such compensation illusions might influence the post-stage processing of seismic data..

Since previous scholars did not consider the influence of marine channel on geometrical spreading compensation, this paper studies how to quickly and accurately conduct amplitude compensation under the deep-water condition. First, the basic principle of 2.5-D geometrical spreading compensation is introduced. Then, the seawater velocity changes of the marine deep-water model are analyzed. Based on research findings of previous scholars, the variable seawater velocity model is adopted for 2.5-D geometrical spreading compensation to verify the accuracy and validity of this method. At last, this paper gives the calculation example with the field data.

\section{Theory}

In order to realize the 2.5-D geometrical spreading compensation technique, the geometrical spreading compensation formula should be built first. Geometrical spreading refers to geometric divergency. The farther the wave spreads, the more wave energy is spread. In the homogeneous medium, the energy density and the square of the wave travel distance is inversely proportional to each other. The elastic waves have several reflections and transformations in the elastic medium of the level course. When there is one turning point at most, the amplitude of the received wave is proportion to the following formula(Ursin, 1990; Sun, 1996; Sun, 2000):

$$
A=\frac{v_{s}}{\mathrm{~L}(g, s)} \mathrm{A} R_{C}
$$

Where, $R_{C}$ stands for the reflection coefficient of the reflection point in $R$; A stands for the total transmission loss when the ray passes through the interface; $\mathrm{L}(g, s)$ stands for the relative geometrical spreading compensation factor.

According to the ray theory, in the homogenous layered medium, it is assumed that the ray, $\Omega$, generated by $s$ is spread to the receiving point, $g ; \mathrm{L}^{\square}(g, s)$ and $\mathrm{L}^{\perp}(g, s)$ are called relative geometrical spreading within and outside the plane, respectively, (the two are reciprocal ). To the 2-D line source, the ray spreads only in the 2-D plane. The geometrical spreading outside the plane is $\mathrm{L}^{\perp}(\mathrm{g}, s)=1$. In terms of the point source, below is the expression formula:

$$
\mathrm{L}^{\square}(g, s)=\left|Q_{2}^{\square}(g, s)\right|^{1 / 2}=\left|\cos \theta_{1} \cos \theta_{n}(\partial x / \partial p)\right|^{1 / 2}
$$




$$
\mathrm{L}^{\perp}(g, s)=\left|Q_{2}^{\perp}(g, s)\right|^{1 / 2}=|\sigma(g, s)|^{1 / 2}=\left(\int_{s}^{g} v d s\right)^{1 / 2}=\left|\frac{x}{p}\right|^{1 / 2}
$$

$x$ stands for the horizontal distance between $s$ and $g ; \theta_{n}$ stands for the angle of incidence of the $n$ layer; $p=\sin \theta_{n} / v_{n}$ stands for the ray parameter; $\mathbf{Q}_{2}$ stands for the submatrix of ray spreading matrix.

In the vertical inhomogeneous medium, $x$ meets the condition of the following ray equation in terms of any ray section:

$$
x=\int_{z_{0}}^{z} \frac{p v d z}{\left(1-p^{2} v^{2}\right)^{1 / 2}}
$$

Where, $z$ stands for the underground medium burial depth. The expression of the relative geometrical spreading factor is shown below:

$$
\mathrm{L}_{n}(g, s)=\mathrm{L}^{\square}(g, s) \mathrm{L}^{\perp}(g, s)=\left(\cos \theta_{1} \cos \theta_{n} \int_{z_{0}}^{z} \frac{v d z}{\left(1-p^{2} v^{2}\right)^{1 / 2}} \int_{z_{0}}^{z} \frac{v d z}{\left(1-p^{2} v^{2}\right)^{3 / 2}}\right)^{1 / 2}
$$

If the source and the receiving point are in the same stratum when the ray is reflected in the medium, the equation is as follows:

$$
\mathrm{L}_{2 n}(g, s)=2 \cos \theta_{1}\left(\int_{z_{0}}^{z} \frac{v d z}{\left(1-p^{2} v^{2}\right)^{1 / 2}} \int_{z_{0}}^{z} \frac{v d z}{\left(1-p^{2} v^{2}\right)^{3 / 2}}\right)^{1 / 2}
$$

Assuming that $\cot \theta \rightarrow 1$, we can get the relative spreading factor of the zero shotoffset:

$$
\mathrm{L}(s=g)=\int_{z_{0}}^{z} v d z
$$

To sum up, Eq.6 and Eq.7 are the relative geometrical spreading compensation factor of 2.5-D non-zero shot-offset and the zero shot-offset. During practical applications, compared with the issue of the 2-D line source, the issue of geometrical spreading generated by a point source in the plane interest the researchers more. This issue is in essence an issue related to 2.5-D. The geometrical spreading of 2.5-D and the standard $2-\mathrm{D}$ is the same when the wave propagates within the 2-D plane. The only difference is whether geometrical spreading outside the plane has been considered. Under the general 2-D conditions, there is no geometrical spreading outside the plane. However, geometrical spreading outside the plane should be conformed to the data outside the plane. Therefore, a 3-D observation system is required. In fact, the integral of $\sigma(g, s)$ through the ray can be worked out to conduct a simple and approximate confirmation of geometrical spreading outside the plane. From the numerical perspective, the difference between 2-D and 2.5-D lies in whether the integral of $\sigma(g, s)$ is calculated along the ray. Just to be equivalent to a 3-D calculation conducted in a 2-D model. This not only 
meets the calculation of amplitude under the 2-D conditions, but also effectively reduces the computational amount. Thus, it is of vital importance to improving the realtime software interaction processing speed of data.

\section{Synthetic data analysis}

The modern marine oil exploration has the following characteristics: 1) Seismic exploration through long shot offset; 2) Thick seawater; 3) Non-ignorable influence of marine channel on spreading velocity of seismic wave. Thus, during the geometrical spreading compensation of the seismic data, these characteristics should be taken into full consideration. In other words, the conventional zero shot-shot offset compensation methods cannot meet requirements of the modern marine seismic data processing. In view of the problems, 2.5-D geometrical spreading compensation is adopted for amplitude restoration.

Under general conditions, the seawater velocity is usually assumed to be constant as $1,500 \mathrm{~m} / \mathrm{s}$ during the practical processing of marine seismic data. However, the research of Han et al. in 2012 suggested that from the perspective of Physical Oceanography the spreading velocity of acoustic waves in the seawater relies on seasons, time, positions, water depth and ocean currents. The major influencing factors are temperature, salinity and pressure. In China's South China Sea area, the temperature variation range is $0<\mathrm{T}$ $<30^{\circ} \mathrm{C}$ in the first $1,000 \mathrm{~m}$. The temperature is relatively high near the sea level. After 1,000 under the sea, the temperature remains basically the same at around $0^{\circ} \mathrm{C}$. The pressure variation range is $1<\mathrm{P}<300 \mathrm{~kg} / \mathrm{cm}^{2}$. The salinity variation range is $33<\mathrm{S}<$ 37 (\%). In this way, the velocity variation curve can be obtained. (See Fig. 1) Considering the non-ignorable influence of seawater velocity variation on ray path, seismic travel time and amplitude, theories of Physical Oceanography can be adopted to optimize the seawater velocity model partially so as to get the optimal amplitude restoration.

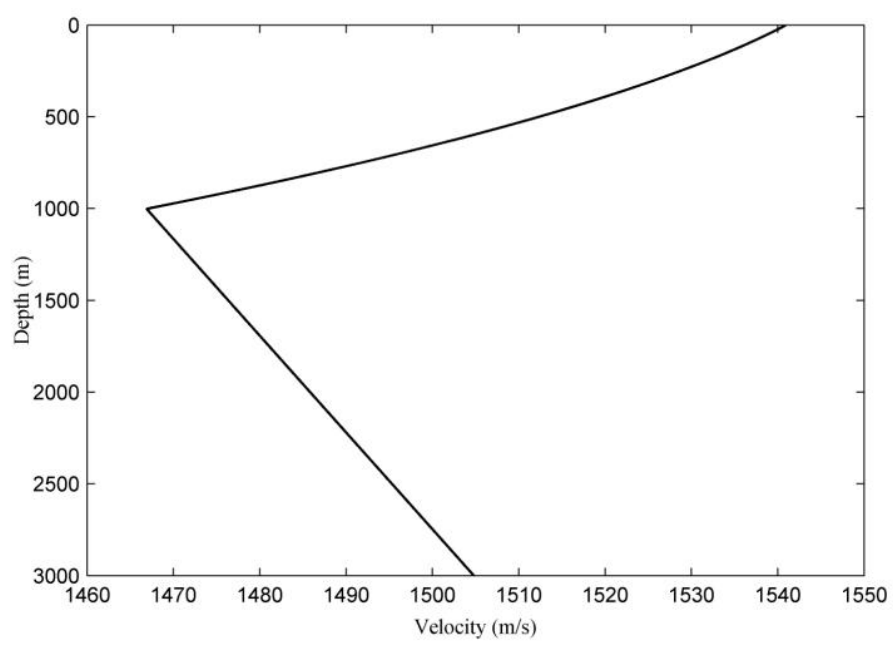

Figure 1. The change of the seismic wave velocity with depth in the seawater.

From the curve variation rules shown in Fig. 1, it can be seen that the seawater velocity has an obvious inflection point at the seawater depth of $1,000 \mathrm{~m}$. This is caused 
by the influence of marine channel on the seawater velocity. The seawater velocity decreases in the shallow layer and then increases in the deep layer.

In order to analyze the difference between the conventional compensation and the 2.5-D compensation, the influence of the marine channel is considered. Two layered medium models, namely the seawater with the constant seawater velocity and the optimized seawater velocity (refer to Table 1 and Table 2 for the velocity models), are adopted for numerical simulation and analysis. Table 1 and Table 2 provide the velocity of various layers and the two-way travel time of each corresponding primary reflection wave. In the model with the optimized seawater velocity, the seawater depth of $1,000 \mathrm{~m}$ is adopted as a boundary line. The seawater layer is divided into two. The average velocity is used to replace the seawater velocity variations so as to get a more accurate seawater velocity model. The shot point and the receiver point are put on the sea surface. Left blasting is adopted. There are 600 channels totally. The shot-receiver offset is $200 \mathrm{~m}$; the channel interval is $10 \mathrm{~m}$; the sampling interval is $1 \mathrm{~ms}$; the number of sampling points is 7,000. Through the two models mentioned above, we compare differences of compensation factors of different compensation methods.

Table 1. The velocity model 1 with constant seawater velocity

\begin{tabular}{c|c|c|c|c|c}
\hline level & $\mathbf{1}$ & $\mathbf{2}$ & $\mathbf{3}$ & $\mathbf{4}$ & $\mathbf{5}$ \\
\hline Velocity (m/s) & 1500 & 2000 & 2400 & 3200 & 3500 \\
Two-way time (s) & 4 & 4.5 & 4.917 & 5.542 & 6.113 \\
\hline
\end{tabular}

Table 2. The velocity model 2 with optimized seawater velocity

\begin{tabular}{c|c|c|c|c|c|c}
\hline level & $\mathbf{1}$ & $\mathbf{2}$ & $\mathbf{3}$ & $\mathbf{4}$ & $\mathbf{5}$ & $\mathbf{6}$ \\
\hline Velocity (m/s) & 1509.5 & 1485.8 & 2000 & 2400 & 3200 & 3500 \\
\hline Two-way time (s) & 1.325 & 4.017 & 4.517 & 4.934 & 5.559 & 6.130
\end{tabular}

During the marine data processing process, the compensation value of the 2-D zero shot-offset compensation method and the 2.5-D geometrical spreading compensation in the constant seawater velocity and the optimized seawater model, respectively, are obviously different.

From Fig. $2 a$, it can be seen that the relative error between the 2.5-D compensation and the conventional compensation is extremely huge. When the offset is small, such error is within the tolerable range. However, with the increase of the offset, the relative error even exceeds by more than $200 \%$. This suggests that under the condition that offset is small and certain error is tolerable the conventional geometrical spreading compensation methods are quick and convenient compensation methods. However, in terms of compensation under the condition of a large offset, the error is huge. From Fig. $2 b$, it can be seen that the relative error of compensation factors is obvious when offset is huge. All relative errors are below $11 \%$. Some relative errors are above 5\%. This indicates the hypothesis of the constant seawater velocity widely adopted at present is reasonable. However, it also shows that changes of compensation factors caused by the influence of marine channel on the ray path, seismic travel time and amplitude are obvious. 


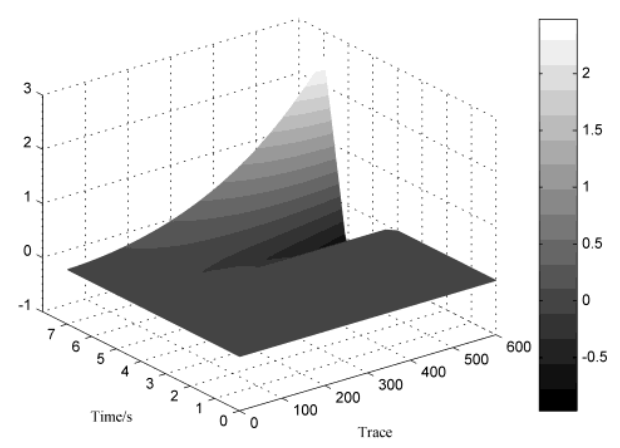

(a)

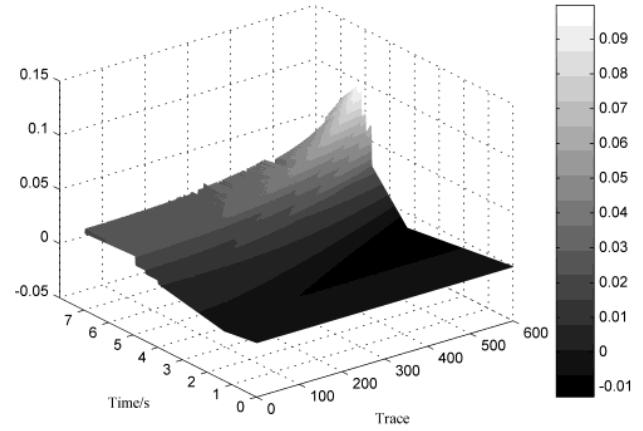

(b)

Figure 2. (a)Percentage of contrast between 2.5-D geometrical spreading compensation factor and zero shot-offset distance compensation factor in the constant seawater velocity.(b) Percentage of contrast between the constant and the optimized seawater velocity with 2.5-D geometrical spreading compensation.

From Fig. $3 a$, it can be seen that the influence of the marine layer on compensation factors can almost be ignored through compensation factors comparison in the 600th channel, and is almost in line with the compensation curve of the constant seawater velocity. Since the travel time of various reflection layers to each receiver is different, the constant seawater velocity compensation curve is first smaller and then larger than the compensation value after $4 \mathrm{~s}$.

Fig. $3 b$ shows the compensation value comparison of the 6,000th sampling point. The compensation value under the condition of zero shot-offset must be a constant value in certain sampling point. The influence of the seawater layer on the compensation factors is shown in Fig. $3 a$ obviously. The compensation value increases along with the shot-offset gradually, and then decreases around the 400th channel, because the bottom reflection has not yet reached the receiver far away.

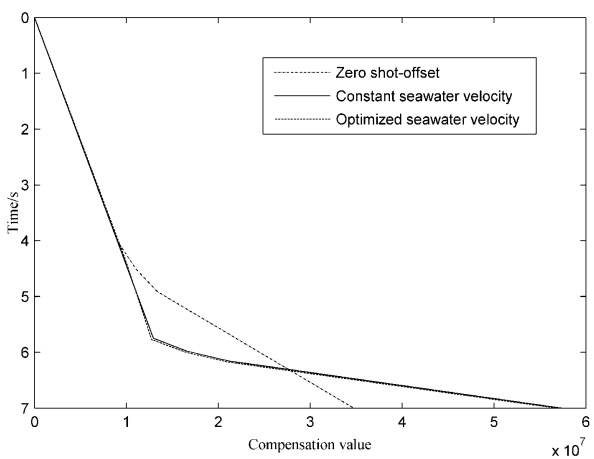

(a)

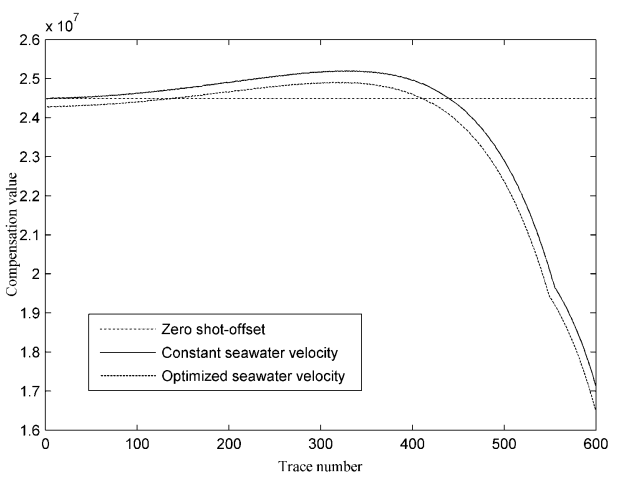

(b)

Figure 3. Comparison among zero shot-offset compensation factor and 2.5-D compensation factor in constant seawater velocity and in optimized seawater velocity:(a)Comparison diagram of compensation values in trace 600th.(b)Comparison diagram of compensation values in the 6000th sampling point. 
Fig. 3 shows the comparison of different channels and sampling points. The 2.5-D optimized seawater velocity compensation curve and the compensation curve under the condition of zero-offset and constant seawater velocity are obvious different from each other. The zero-offset compensation and the 2.5-D constant seawater velocity compensation cannot accurately realize the compensation of various layers. However, the 2.5-D optimized seawater can make it and is more suitable for geometrical spreading compensation of long-offset data.

Fig. 4 provides the compensation factors of the 2.5-D constant seawater velocity and optimized seawater velocity compensation factors along the reflecting interface. As is shown, seawater channel can exert an influence on the seawater velocity. Consequently, compensation factors of various reflecting layers are influenced. Besides, the influence is augmented due to a long offset. The influence of marine channel on the compensation value increases along with the increase of offset.

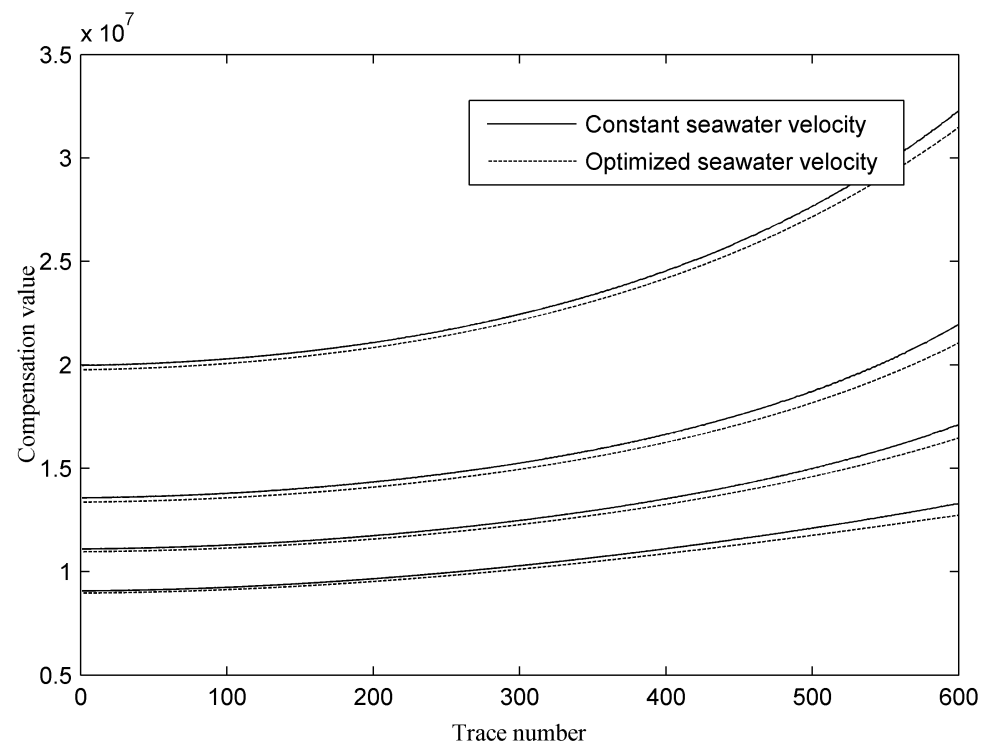

Figure 4. Compensation values at the arriving time from reflectors in the case of 2.5-D compensation factor in constant seawater velocity and in optimized seawater velocity.

\section{Field data processing}

In order to verify the correctness and reasonability of the method put forward in this paper, we choose field data of a sea for geometrical spreading compensation.

The original seismic records of common shot gathers are shown in Fig. 5a. It can be seen that the energy in the shallow layer is relatively huge, easy to recognize and abundant in information; but the attenuation in the deep layer is fast, and the seismic information cannot be recognized. First, amplitude restoration is conducted of the original seismic records of common shot gathers through the conventional geometrical spreading. From Fig. 5b, it can be clearly seen that the deep-layer energy has achieved favorable restoration. The seismic information has been enriched, but the energy restoration in the long shot-offset is not optimistic. In terms of energy equilibrium, the energy in the shallow and deep layer does not completely match each other. This suggests that the amplitude energy compensation is uneven and inaccurate. 


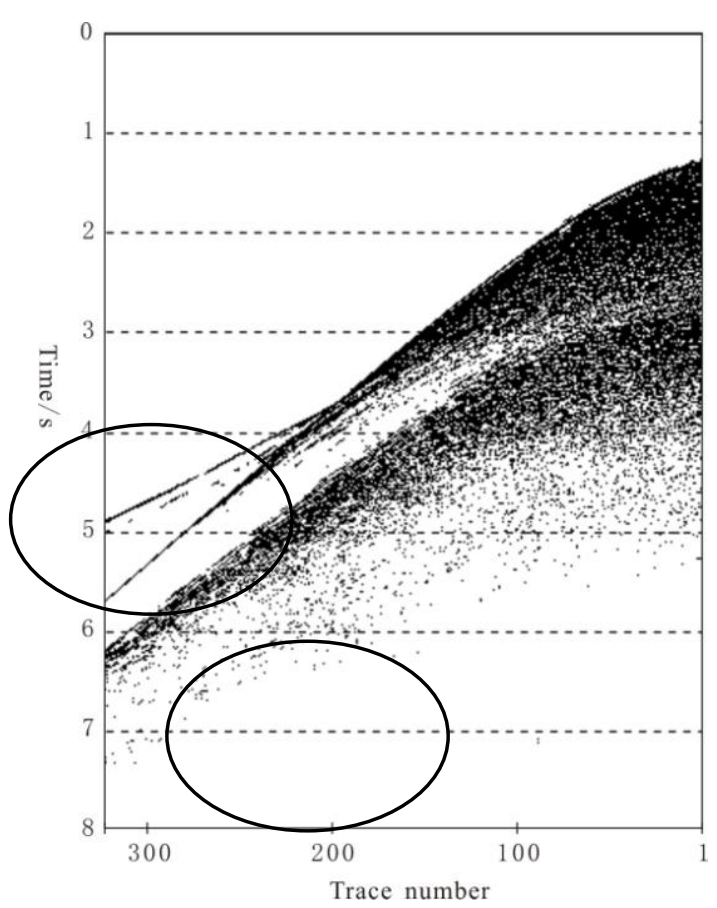

(a)

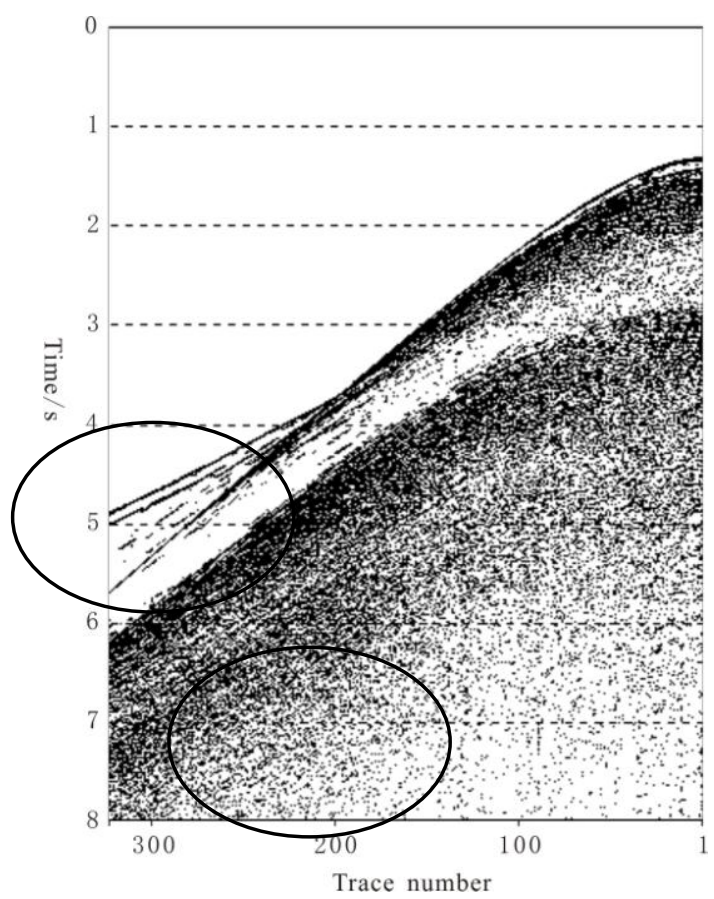

(c)

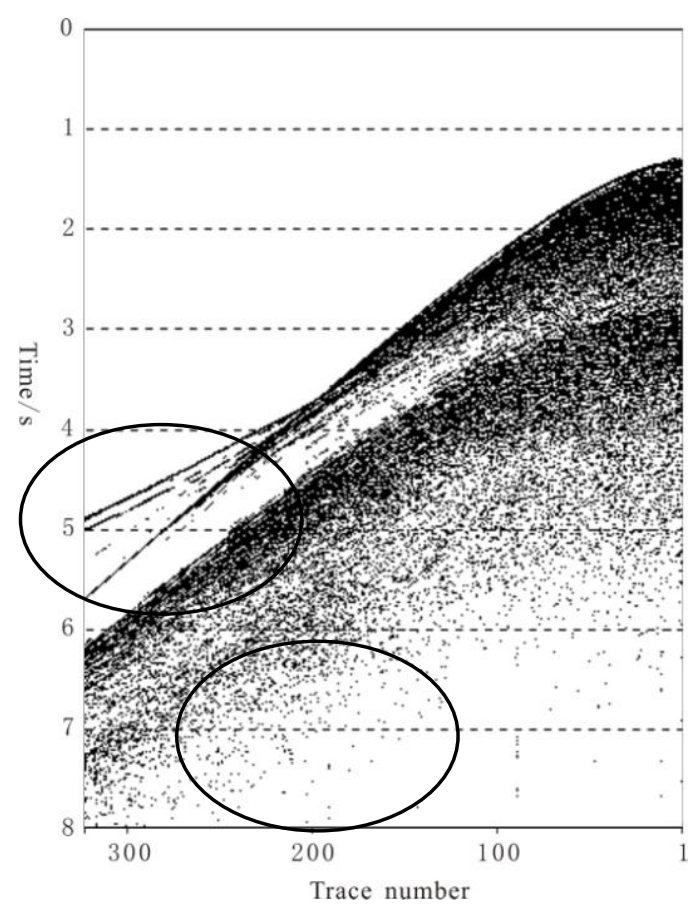

(b)

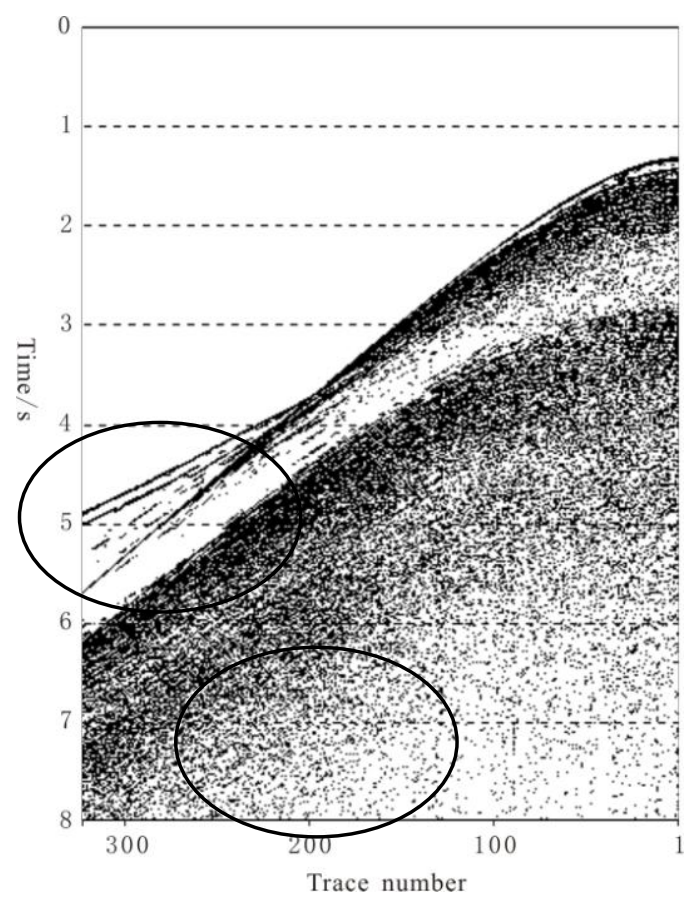

(d)

Figure 5. Comparison diagrams of seismic record after applying geometrical spreading compensation in different methods: (a) initial seismic record,(b)seismic record that compensated by zero shot-offset compensation factor $(c)$ seismic record that compensated by 2.5-D compensation factor with constant seawater velocity $(d)$ seismic record that compensated by 2.5-D compensation factor with variable seawater velocity

Fig. 5c and Fig. 5d show the 2.5-D geometrical spreading compensation of seismic records of common shot gathers in the model with the constant seawater velocity and 
the optimized seawater velocity. The seismic records after 2.5 -D compensation show that the energy either in the near and long shot-offset or the deep and shallow layer is more balanced than that in Fig. 5b. Basically speaking, the seismic amplitude is restored completely. Compared with Fig. $5 c$ in terms of energy, Fig. $5 d$ shows that the energy in the shallow layer is slightly stronger, but weaker in the deep layer. However, the whole records are more balanced and reasonable, because the influence of the seawater layer on the ray tracing and geometrical spreading compensation is considered.

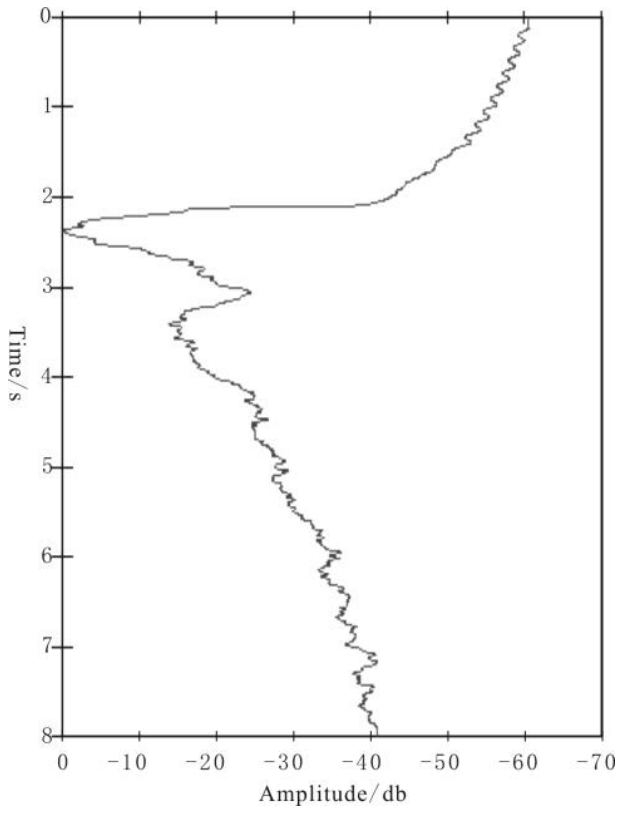

(a)

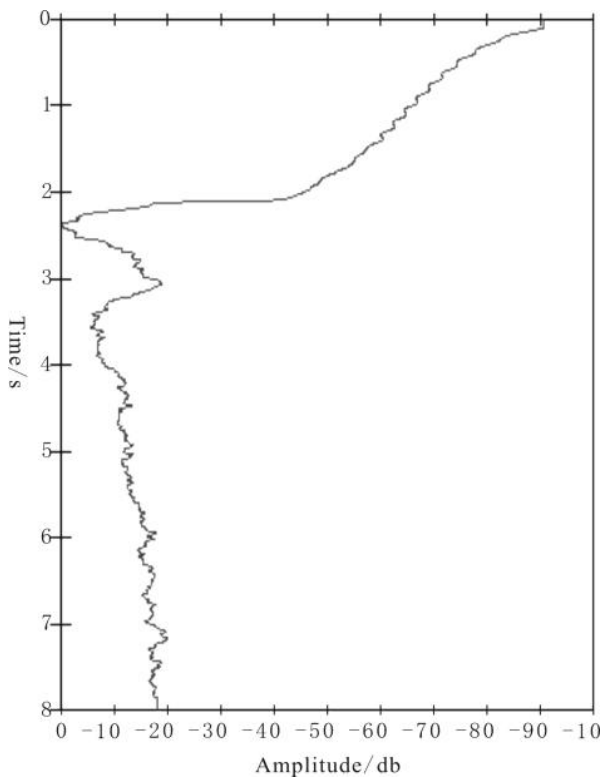

(c)

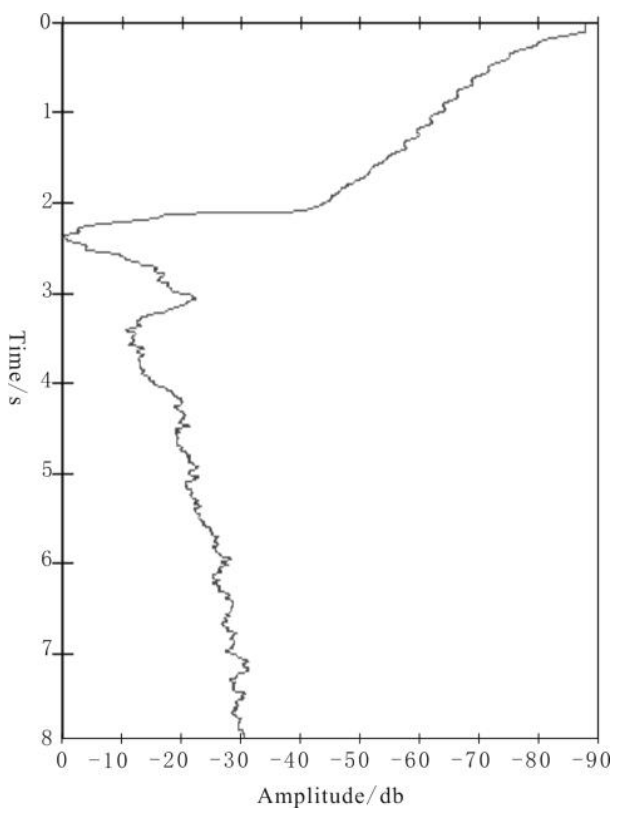

(b)

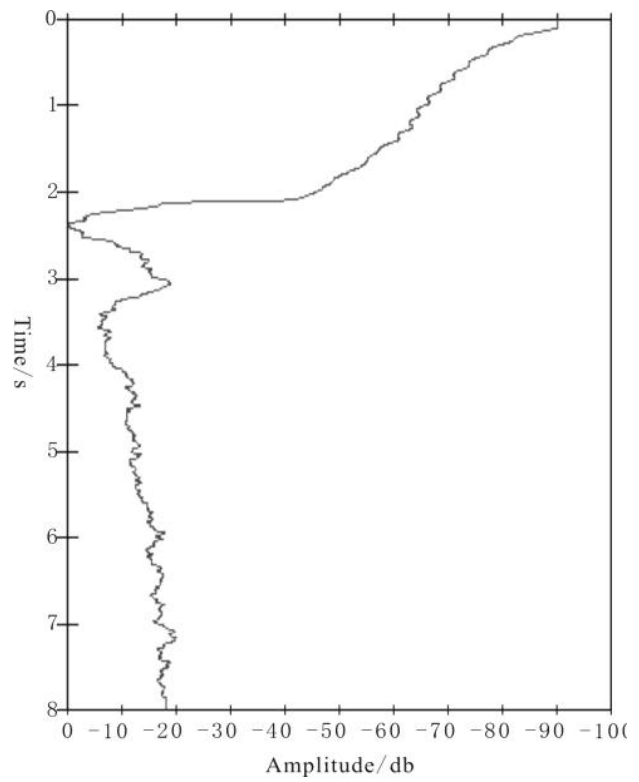

(d)

Figure 6. Comparison diagrams of the No.100 trace amplitudes in seismic records corresponding to Fig.5:(a)initial seismic record,(b)compensated by zero shot-offset compensation factor, (c) compensated by 2.5-D compensation factor with constant sea velocity, $(d)$ compensated by 2.5-D compensation factor with variable sea velocity 
Fig. 6 shows the amplitude curve of the 100th channel corresponding to the seismic records in Fig. 5. From Fig. 6, it can be directly seen that the amplitude curve under the zero-offset compensation registers a huge improvement in terms of equilibrium compared with the original amplitude curve. Its energy value is more balanced under the seabed interface. This suggests that the zero offset compensation can achieve preliminary compensation. However, compared with the zero-offset compensation results, the energy equilibrium effect after 2.5-D compensation is improved more than two times. The amplitude curve of 2.5-D seawater compensation is slightly superior to the amplitude curve under the 2.5-D constant seawater velocity, but the difference is not huge generally speaking. This is related to the seawater depth. When seismic data in the deeper seawater layer calls for compensation, the influence of marine channel becomes extremely significant. From Fig. 6, 2.5-D compensation can achieve a more accurate restoration of amplitude.

\section{Conclusions}

Proceeding from the perspective of Physical Oceanography, this paper studies the theoretical models and analyzes the influence of marine channel and offset on the geometrical spreading compensation value. Concerning the long offset and deep water conditions during the geophysical exploration process, this paper puts forward the 2.5-D geometrical spreading compensation technique to conduct more accurate amplitude restoration of the horizontal layered or seemingly horizontal layered medium. The method fully considers the geometrical spreading compensation and the marine channel outside the plane in the 2-D observation system to achieve optimal restoration of the amplitude. During the processing of the practical marine seismic data, the following problems were encountered:

1) The reflecting wave generated by different reflecting layers is recorded by the receiver in different time. When the offset of the practical marine seismic exploration data is long, the reflection record might be interacted with each other in seismic records in the long shot. Similarly, the compensation factors about the specific reflecting layer worked out in advance are also overlapped. Considering the conditions of the intersection point of reflecting record, we adopts a larger compensation value as the final compensation value of the point.

2) The seismic signals are made up of reflection records and seismic wavelets. The seismic wavelets have certain duration length. As a result, during geometrical spreading compensation of seismic records, it is necessary to compensate every wavelet in the reflection records and ensure that the compensation value is the same. Generally speaking, the duration length of a seismic wavelet is $200 \mathrm{~ms}$.

3) Marine seismic exploration data usually cover large scale of fields. The special marine sedimentary environment has resulted in turning the underground medium into the layered medium or seemingly layered medium in a small area (such as within the single shot scope). In a huge scope, the underground medium might be the rugged seabed or the steep angle or other special conditions. In my opinion it is feasible to subdivide the velocity model of the whole measuring line by dividing the part with slow velocity variation into one area and the area such as the rugged seabed or the steep angle or other harsh conditions into multiple small areas. In this way, various areas after division can be approximately regarded as the layered medium for 2.5-D geometrical spreading compensation. 
4) After marine seismic data collection in certain sea area, we guess that it is necessary to consider partial seawater velocity distribution obtained through physical oceanographical methods, and find the feasible seawater velocity model according to the position of marine channel so as to achieve proper geometrical spreading compensation.

During the processing of practical marine seismic data, the above problems encountered are solved. By comparing with the conventional compensation methods, the accuracy and reasonability of the $2.5-\mathrm{D}$ compensation method are verified. The method put forward in this paper improves the reliability of amplitude restoration.

Acknowledgements. This study was supported financially by the National Natural Science Foundation of China (41274120, 41404085, 41504084).

\section{REFERENCES}

[1] Bleistein, N.(1986): Two-and-one-half Dimensonal In-plane Wave Propagation. Geophysical Prospecting 34(5): 686-703.

[2] Bortfeld, R.(1989): Geometrical ray theory: Rays and traveltimes in seismic systems (second-order approximations of the traveltimes). - Geophysics 54(3): 342-349.

[3] Červený, V.(2001): Seismic ray theory. - Cambridge University Press.

[4] Červený, V., Ravindra, R.(1971): Theory of seismic head waves. University of Toronto Press.

[5] Ettrich, N.(2002): Offset-dependent geometrical spreading in isotropic laterally homogeneous media using constant velocity gradient models. - Geophysics 67(5): 16121615.

[6] Han, F. X., Sun, J. G., Wang, K.(2012).: The influence of sea water velocity variation on seismic traveltimes, ray paths, and amplitude. - Applied Geophysics 9(3): 319-325.

[7] Newman, P.(1973): Divergence Effects In A Layered Earth. -Geophysics 38(3): 481-488.

[8] Stovas, A., Ursin, B.(2009): Improved geometric-spreading approximation in layered transversely isotropic media. - Geophysics 74(5): D85-D95.

[9] Sun, J. G.(1996): The relationship between the first Fresnel zone and the normalized geometrical spreading factor. - Geophysical Prospecting 44(3): 351-374.

[10] Sun, J. G.(1998): On the limited aperture migration in two dimensions. - Geophysics 63(3): 984-994.

[11] Sun, J. G.(2000): Limited-aperture migration. - Geophysics 65(2): 584-595.

[12] Tygel, M., Schleicher, J., Hubral, P.(1992): Geometrical spreading corrections of offset reflections in a laterally inhomogeneous Earth. - Geophysics 57(8): 1054-1063.

[13] Ursin, B.(1978): Wavefront Curvature In A Layered Medium. - Geophysics 43(5): 10111013.

[14] Ursin, B.(1990): Offset-dependent geometrical spreading in a layered medium. Geophysics 55(4): 492-496.

[15] Ursin, B., Dahl, T.(1992): Seismic Reflection Amplitudes. - Geophysical Prospecting 40(5): 483-512.

[16] Ursin, B., Hokstad, K.(2003): Geometrical spreading in a layered transversely isotropic medium with vertical symmetry axis. - Geophysics 68(6): 2082-2091.

[17] Ursin, B., Stovas, A.(2006): Traveltime approximations for a layered transversely isotropic medium. - Geophysics 71(2): D23-D33. 